\title{
On Products and Mixed Sums of Gamma and Beta Random Variables Motivated by Availability
}

DOI:

10.1007/s11009-017-9591-2

\section{Document Version}

Accepted author manuscript

Link to publication record in Manchester Research Explorer

\section{Citation for published version (APA):}

Homei, H., \& Nadarajah, S. (2017). On Products and Mixed Sums of Gamma and Beta Random Variables Motivated by Availability. Methodology and Computing in Applied Probability, 1-12. https://doi.org/10.1007/s11009017-9591-2

\section{Published in:}

Methodology and Computing in Applied Probability

\section{Citing this paper}

Please note that where the full-text provided on Manchester Research Explorer is the Author Accepted Manuscript or Proof version this may differ from the final Published version. If citing, it is advised that you check and use the publisher's definitive version.

\section{General rights}

Copyright and moral rights for the publications made accessible in the Research Explorer are retained by the authors and/or other copyright owners and it is a condition of accessing publications that users recognise and abide by the legal requirements associated with these rights.

\section{Takedown policy}

If you believe that this document breaches copyright please refer to the University of Manchester's Takedown Procedures [http://man.ac.uk/04Y6Bo] or contact uml.scholarlycommunications@manchester.ac.uk providing relevant details, so we can investigate your claim.

\section{OPEN ACCESS}


1. 


\title{
On products and mixed sums of gamma and beta random variables motivated by availability
}

\author{
Hazhir Homei and Saralees Nadarajah \\ Department of Statistics, University of Tabriz, Tabriz, IRAN \\ School of Mathematics, University of Manchester, Manchester, UK
}

November 7, 2017

\begin{abstract}
Motivated by the notion of availability, the exact distribution of the sum of beta random variables is derived under the impact of the environment. A suitable approximation is presented when the exact distribution cannot be identified. Also presented are some characterizations for the gamma distribution.
\end{abstract}

Keywords: Availability; Beta distribution; Lifetime.

\section{Introduction}

The distribution of a linear combination of random variables arises in many applied problems. It has been extensively studied by different researchers. The distribution of linear combinations of the form $\alpha X+\beta Y$ is of interest in problems in automation, control, fuzzy sets, quality, reliability engineering, and other areas of computer science (see e.g. [15, 23] and the references therein). There has been a great interest in the study of the distributions of the linear combination $\alpha X+\beta Y$ when $X$ and $Y$ are random variables and belong to beta families, see e.g. [22], [2], [3] and [4]. Some systems are subjected to a wide variety of fluctuations and interruptions; varying weather conditions, learning development on repetitive operations, equipment breakdowns, management interference, and other external factors may impact the output.

In the systems mentioned above, it is quite possible that $\alpha$ and $\beta$ could be random variables themselves. The distribution of the stochastic linear combination (see Definition 2.2 in [11]) is of interest in engineering. Measuring attributes in different fields in engineering may be necessary. Also, a suitable statistical analysis of these attributes can be useful in decision making. These attributes are measured usually in laboratories in ideal conditions, which can be different from the usual observations in practice. Lifetime, availability, vehicle speed, etc are all scientific problems investigated in ideal conditions. But in most papers the environmental conditions are ignored. As an example, survival analysis is of fundamental importance in quality technology, see e.g. [5], [7], [8], [20] and [6]. Usually, lifetimes measured in a laboratory are different observations by different users in the environment. Suppose that $Y_{i}$ 's are the lifetimes measured in a laboratory and $X_{i}$ (with $0 \leq X_{i} \leq 1$ ) are the random effects of the environment on the lifetimes, such that $X_{i} Y_{i} \leq Y_{i}$ holds, and so $\sum_{i} X_{i} Y_{i}$ is the total lifetime in the environment. Here, we find the distribution of the sum of beta random variables under environmental impact. The innovative and interesting 
works of some authors (see [14] and Theorem 2.2) lead us to using some suitable approximations for distributions of these categories of random variables.

\section{Total availability under environmental impact}

The concept of availability ${ }^{1}$ and [1] motivate us to discuss the distribution of the sum of beta random variables in this section. The method of this section for computing the exact distribution is a continuation of the method of [26, Section 7]. Throughout, $\left(W_{1}, \ldots, W_{r}\right)$ is called random coefficient vector of environmental effect in $r$-position. The following theorem can be proved straightforwardly.

Theorem 2.1. Suppose $X_{1}, \ldots, X_{r}$ are independent random variables having, respectively, the $\operatorname{Beta}\left(n_{1}, m_{1}\right), \ldots, \operatorname{Beta}\left(n_{r}, m_{r}\right)$ distributions and that the random vector $W=\left\langle W_{1}, \ldots, W_{r}\right\rangle$ has the Dirichlet $\left(\alpha_{1}, \ldots, \alpha_{r}\right)$ distribution. Then the moments of $Z=\sum_{j=1}^{r} W_{j} X_{j}$ are

$$
E\left(Z^{k}\right)=\sum_{i_{1}+\cdots+i_{r}=k} k \operatorname{Beta}\left(\sum_{j=1}^{r} \alpha_{j}, k\right) \prod_{j=1}^{r} \frac{\operatorname{Beta}\left(n_{j}+m_{j}, \alpha_{j}+i_{j}\right)}{i_{j} \operatorname{Beta}\left(n_{j}, i_{j}\right) \operatorname{Beta}\left(m_{j}+n_{j}+i_{j}, \alpha_{j}\right)} .
$$

In particular, the first moment of $Z$ is

$$
E(Z)=\frac{\sum_{i=1}^{r} \alpha_{i} n_{i} /\left(n_{i}+m_{i}\right)}{\sum_{i=1}^{r} \alpha_{i}}
$$

The mean, variance and entropy of $Z$ for different values of parameters are given in Table 1 .

\footnotetext{
${ }^{1}$ See e.g. the ARES conference series: https://www.ares-conference.eu/
} 
Table 1: The mean, variance, and entropy.

\begin{tabular}{|c|c|c|c|c|c|c|}
\hline$\left(n_{1}, m_{1}\right)$ & $\left(n_{2}, m_{2}\right)$ & $\left(n_{3}, m_{3}\right)$ & $E(Z)$ & $E\left(Z^{2}\right)$ & $\operatorname{Var}(Z)$ & entropy \\
\hline$(1,1)$ & $(1,1)$ & $(1,1)$ & 0.5 & 0.3079 & 0.0579 & -0.0716 \\
$(1,1)$ & $(1,1)$ & $(1,1)$ & 0.5 & 0.291 & 0.041 & -0.1998 \\
$(1,1)$ & $(1,1)$ & $(1,1)$ & 0.5 & 0.287 & 0.037 & -0.2417 \\
$(1,1)$ & $(1,1)$ & $(1,1)$ & 0.5 & 0.283 & 0.033 & -0.2993 \\
$(1.1)$ & $(1,1)$ & $(1,1)$ & 0.5 & 0.28 & 0.030 & -0.3349 \\
$(1,1)$ & $(1,1)$ & $(1,1)$ & 0.5 & 0.283 & 0.033 & -0.3005 \\
$(2,6)$ & $(3,7)$ & $(4,8)$ & 0.303 & 0.105 & 0.013191 & -0.7583 \\
$(2,6)$ & $(3,7)$ & $(4,8)$ & 0.294 & 0.096 & 0.009564 & -0.9103 \\
$(2,6)$ & $(3,7)$ & $(4,8)$ & 0.3008 & 0.0991 & 0.008619 & -20.8889 \\
$(2,6)$ & $(3,7)$ & $(4,8)$ & 0.303 & 0.099 & 0.007191 & -1.0443 \\
$(2,6)$ & $(3,7)$ & $(4,8)$ & 0.301 & 0.0978 & 0.007199 & -1.0821 \\
$(2.6)$ & $(3,7)$ & $(4,8)$ & 0.3083 & 0.1022 & 0.007151 & -1.059 \\
$(3,11)$ & $(5,13)$ & $(7,15)$ & 0.5 & 0.307 & 0.057 & -0.0716 \\
$(3,11)$ & $(5,13)$ & $(7,15)$ & 0.27 & 0.078 & 0.0051 & -1.18 \\
$(3,11)$ & $(5,13)$ & $(7,15)$ & 0.278 & 0.082 & 0.004716 & -1.2484 \\
$(3,11)$ & $(5,13)$ & $(7,15)$ & 0.281 & 0.083 & 0.004039 & -1.3318 \\
$(3,11)$ & $(5,13)$ & $(7,15)$ & 0.279 & 0.081 & 0.003159 & -1.3736 \\
$(3,11)$ & $(5,13)$ & $(7,15)$ & 0.287 & 0.086 & 0.003631 & -1.352 \\
$(4,16)$ & $(7,19)$ & $(10,22)$ & 0.273 & 0.080 & 0.05471 & -1.1631 \\
$(4,16)$ & $(7,19)$ & $(10,22)$ & 0.260 & 0.072 & 0.0044 & -1.338 \\
$(4,16)$ & $(7,19)$ & $(10,22)$ & 0.269 & 0.075 & 0.002639 & -1.4141 \\
$(4,16)$ & $(7,19)$ & $(10,22)$ & 0.272 & 0.077 & .003016 & -1.5081 \\
$(4,16)$ & $(7,19)$ & $(10,22)$ & 0.27 & 0.075 & 0.0021 & -1.554 \\
$(4,16)$ & $(7,19)$ & $(10,22)$ & 0.279 & 0.080 & 0.02159 & -1.1106 \\
$(5,21)$ & $(9,25)$ & $(13,29)$ & 0.268 & 0.076 & 0.004176 & -1.5345 \\
$(5,21)$ & $(9,25)$ & $(13,29)$ & 0.255 & 0.068 & 0.002975 & -1.4463 \\
$(5,21)$ & $(9,25)$ & $(13,29)$ & 0.264 & 0.072 & 0.002304 & -1.5299 \\
$(5,21)$ & $(9,25)$ & $(13,29)$ & 0.268 & 0.074 & 0.002176 & -1.6342 \\
$(5,21)$ & $(9,25)$ & $(13,29)$ & 0.265 & 0.072 & 0.001775 & -1.6839 \\
$(5,21)$ & $(9,25)$ & $(13,29)$ & 0.275 & 0.077 & 0.001375 & -1.667 \\
\hline
\end{tabular}


Table 2: Checking the robustness of the approximation for $r=2$.

\begin{tabular}{|c|c|c|c|c|c|c|c|}
\hline$\left(n_{1}, m_{1}\right)$ & $\left(n_{2}, m_{2}\right)$ & $\left(\alpha_{1}, \alpha_{2}\right)$ & $E(Z)$ & $\operatorname{Var}(Z)$ & $p$ & $q$ & $p$-value \\
\hline$(1,1)$ & $(1,1)$ & $(1,1)$ & 0.5 & 0.055 & 1.75 & 1.75 & 0.89 \\
$(1,1)$ & $(1,1)$ & $(0.2,0.3)$ & 0.5 & 0.07 & 1.28 & 1.28 & 0.90 \\
$(1,1)$ & $(1,1)$ & $(1.75,2)$ & 0.5 & 0.05 & 1.97 & 1.97 & 0.38 \\
$(1,1)$ & $(1,1)$ & $(3,5)$ & 0.5 & 0.04 & 2.07 & 2.07 & 0.67 \\
$(1,1)$ & $(1,1)$ & $(7,10)$ & 0.5 & 0.04 & 2.26 & 2.26 & 0.21 \\
$(1,1)$ & $(1,1)$ & $(10,20)$ & 0.5 & 0.04 & 2.13 & 2.13 & 0.35 \\
$(2,4)$ & $(3,5)$ & $(0.2,0.3)$ & 0.36 & 0.3704 & 3.08 & 5.51 & 0.39 \\
$(2,4)$ & $(3,5)$ & $(1.75,2)$ & 0.35 & 0.0175 & 4.31 & 7.82 & 0.54 \\
$(2,4)$ & $(3,5)$ & $(3,5)$ & 0.35 & 0.0175 & 4.75 & 8.47 & 0.46 \\
$(2,4)$ & $(3,5)$ & $(7,10)$ & 0.35 & 0.0175 & 5.05 & 9.07 & 0.12 \\
$(2,4)$ & $(3,5)$ & $(10,20)$ & 0.36 & 0.0104 & 5.0 & 8.85 & 0.47 \\
$(3,7)$ & $(5,9)$ & $(0.2,0.3)$ & 0.33 & 0.0111 & 4.76 & 9.49 & 0.94 \\
$(3,7)$ & $(5,9)$ & $(1.75,2)$ & 0.33 & 0.0011 & 6.64 & 13.45 & 0.65 \\
$(3,7)$ & $(5,9)$ & $(3,5)$ & 0.33 & 0.0111 & 7.42 & 14.69 & 0.28 \\
$(3,7)$ & $(5,9)$ & $(7,10)$ & 0.33 & 0.0111 & 7.87 & 15.72 & 0.46 \\
$(3,7)$ & $(5,9)$ & $(10,20)$ & 0.33 & 0.0111 & 7.89 & 15.44 & 0.80 \\
$(4,10)$ & $(7,13)$ & $(0.2,0.3)$ & 0.32 & 0.0076 & 6.34 & 13.22 & 0.60 \\
$(4,10)$ & $(7,13)$ & $(1.75,2)$ & 0.32 & 0.0066 & 8.91 & 18.94 & 0.38 \\
$(4,10)$ & $(7,13)$ & $(3,5)$ & 0.32 & 0.0076 & 10.066 & 20.82 & 0.29 \\
$(4,10)$ & $(7,13)$ & $(7,10)$ & 0.32 & 0.0076 & 10.67 & 22.31 & 0.22 \\
$(4,10)$ & $(7,13)$ & $(10,20)$ & 0.32 & 0.0076 & 10.76 & 22.007 & 0.91 \\
$(5,13)$ & $(9,17)$ & $(0.2,0.3)$ & 0.31 & 0.0139 & 7.83 & 16.75 & 0.83 \\
$(5,13)$ & $(9,17)$ & $(1.75,2)$ & 0.31 & 0.0039 & 11.12 & 24.27 & 0.31 \\
$(5,13)$ & $(9,17)$ & $(3,5)$ & 0.32 & 0.0056 & 12.66 & 26.86 & 0.53 \\
$(5,13)$ & $(9,17)$ & $(7,10)$ & 0.32 & 0.0056 & 12.66 & 26.85 & 0.75 \\
$(5,13)$ & $(9,17)$ & $(10,20)$ & 0.32 & 0.0066 & 13.63 & 28.53 & 0.97 \\
\hline
\end{tabular}


Table 3: Checking the robustness of the approximation for $r=3$.

\begin{tabular}{|c|c|c|c|c|c|c|}
\hline$\left(n_{1}, m_{1}\right)$ & $\left(n_{2}, m_{2}\right)$ & $\left(n_{3}, m_{3}\right)$ & $\left(\alpha_{1}, \alpha_{2}, \alpha_{3}\right)$ & $p$ & $q$ & $p$-value \\
\hline$(1,1)$ & $(1,1)$ & $(1,1)$ & $(0.2,0.3,0.4)$ & 1.655 & 1.655 & 0.11 \\
$(1,1)$ & $(1,1)$ & $(1,1)$ & $(1,1,1)$ & 2.5 & 2.5 & 0.67 \\
$(1,1)$ & $(1,1)$ & $(1,1)$ & $(1.25,1.75,2)$ & 2.802 & 2.802 & 0.31 \\
$(1,1)$ & $(1,1)$ & $(1,1)$ & $(3,5,6)$ & 3.25 & 3.25 & 0.14 \\
$(1.1)$ & $(1,1)$ & $(1,1)$ & $(7,10,12)$ & 3.55 & 3.55 & 0.44 \\
$(1,1)$ & $(1,1)$ & $(1,1)$ & $(10,20,30)$ & 3.26 & 3.26 & 0.84 \\
$(2,6)$ & $(3,7)$ & $(4,8)$ & $(0.2,0.3,0.4)$ & 4.481 & 10.274 & 0.75 \\
$(2,6)$ & $(3,7)$ & $(4,8)$ & $(1,1,1)$ & 5.949 & 14.257 & 0.78 \\
$(2,6)$ & $(3,7)$ & $(4,8)$ & $(1.25,1.75,2)$ & 0.046 & 16.375 & 0.58 \\
$(2,6)$ & $(3,7)$ & $(4,8)$ & $(3,5,6)$ & 8.365 & 19.191 & 0.80 \\
$(2,6)$ & $(3,7)$ & $(4,8)$ & $(7,10,12)$ & 8.971 & 20.762 & 0.22 \\
$(2.6)$ & $(3,7)$ & $(4,8)$ & $(10,20,30)$ & 8.855 & 19.866 & 0.64 \\
$(3,11)$ & $(5,13)$ & $(7,15)$ & $(0.2,0.3,0.4)$ & 1.655 & 1.655 & 0.11 \\
$(3,11)$ & $(5,13)$ & $(7,15)$ & $(1,1,1)$ & 9.137 & 24.694 & 0.94 \\
$(3,11)$ & $(5,13)$ & $(7,15)$ & $(1.25,1.75,2)$ & 11.092 & 28.798 & 0.61 \\
$(3,11)$ & $(5,13)$ & $(7,15)$ & $(3,5,9)$ & 13.471 & 34.386 & 0.17 \\
$(3,11)$ & $(5,13)$ & $(7,15)$ & $(7,10,12)$ & 14.490 & 37.414 & 0.19 \\
$(3,11)$ & $(5,13)$ & $(7,15)$ & $(10,20,30)$ & 14.543 & 36.061 & 0.17 \\
$(4,16)$ & $(7,19)$ & $(10,22)$ & $(0.2,0.3,0.4)$ & 8.986 & 23.922 & 0.96 \\
$(4,16)$ & $(7,19)$ & $(10,22)$ & $(1,1,1)$ & 11.966 & 33.957 & 0.62 \\
$(4,16)$ & $(7,19)$ & $(10,22)$ & $(1.25,1.75,2)$ & 14.822 & 40.232 & 0.36 \\
$(4,16)$ & $(7,19)$ & $(10,22)$ & $(3,5,6)$ & 018.407 & 49.033 & 0.20 \\
$(4,16)$ & $(7,19)$ & $(10,22)$ & $(7,10,12)$ & 19.912 & 53.722 & 0.38 \\
$(4,16)$ & $(7,19)$ & $(10,22)$ & $(10,20,30)$ & 20.195 & 52.105 & 0.87 \\
$(5,21)$ & $(9,25)$ & $(13,29)$ & $(0.2,0.3,0.4)$ & 10.769 & 22.335 & 0.28 \\
$(5,21)$ & $(9,25)$ & $(13,29)$ & $(1,1,1)$ & 14.486 & 42.209 & 0.53 \\
$(5,21)$ & $(9,25)$ & $(13,29)$ & $(1.25,1.75,2)$ & 18.263 & 50.777 & 0.85 \\
$(5,21)$ & $(9,25)$ & $(13,29)$ & $(3,5,6)$ & 23.170 & 63.157 & 0.71 \\
$(5,21)$ & $(9,25)$ & $(13,29)$ & $(7,10,12)$ & 25.226 & 69.690 & 0.57 \\
$(5,21)$ & $(9,25)$ & $(13,29)$ & $(10,20,30)$ & 25.803 & 68.011 & 0.32 \\
\hline
\end{tabular}

Corollary 2.1. Assume that the random variables $X_{1}, \ldots, X_{r}$ are independent and have the common $\operatorname{Beta}(n, m)$ distribution on $(0,1)$ and the random vector $W=\left\langle W_{1}, \ldots, W_{r}\right\rangle$ has the Dirichlet $\left(\alpha_{1}, \ldots, \alpha_{r}\right)$ distribution. Then the moments of $Z=\sum_{j=1}^{r} W_{j} X_{j}$ are

$$
E\left(Z^{k}\right)=\sum_{i_{1}+\cdots+i_{r}=k} k \operatorname{Beta}\left(\sum_{j=1}^{r} \alpha_{j}, k\right) \prod_{j=1}^{r} \frac{\operatorname{Beta}\left(n+m, \alpha_{j}+i_{j}\right)}{i_{j} \operatorname{Beta}\left(n, i_{j}\right) \operatorname{Beta}\left(m+n+i_{j}, \alpha_{j}\right)} .
$$

The first moment and variance of $Z$ are

$$
E(Z)=\frac{n}{n+m}
$$

and

$$
\operatorname{Var}(Z)=\frac{n m}{(n+m)^{2}(n+m+1)} \frac{\sum_{j=1}^{r} \alpha_{j}\left(\alpha_{j}+1\right)}{\left(\sum_{j=1}^{r} \alpha_{j}\right)\left(\sum_{j=1}^{r} \alpha_{j}+1\right)}
$$


In particular, when $n=m$, we have

$$
E(Z)=\frac{1}{2}
$$

and

$$
\operatorname{Var}(Z)=\frac{1}{4(2 n+1)} \frac{\sum_{j=1}^{r} \alpha_{j}\left(\alpha_{j}+1\right)}{\left(\sum_{j=1}^{r} \alpha_{j}\right)\left(\sum_{j=1}^{r} \alpha_{j}+1\right)} .
$$

Theorem 2.2. Suppose that the independent random variables $X_{1}, \ldots, X_{r}$ have the Beta $\left(n_{1}, m_{1}\right)$, $\ldots, \operatorname{Beta}\left(n_{r}, m_{r}\right)$ distributions and that the random vector $W=\left\langle W_{1}, \ldots, W_{r}\right\rangle$ has the Dirichlet ( $\left.n_{1}+m_{1}, \ldots, n_{r}+m_{r}\right)$ distribution. Then $Z=\sum_{j=1}^{r} W_{j} X_{j}$ has the Beta $\left(\sum_{j=1}^{r} n_{j}, \sum_{j=1}^{r} m_{j}\right)$ distribution.

We find the $k$ th moment of $Z$ as follows:

$$
E\left(Z^{k}\right)=\sum_{i_{1}+\cdots+i_{r}=k} \frac{k !}{i_{1} ! \cdots i_{r} !} E\left(W_{1}^{i_{1}} \cdots W_{r}^{i_{r}}\right) E\left(X_{1}^{i_{1}}\right) \cdots E\left(X_{r}^{i_{r}}\right) .
$$

By using the Dirichlet distribution, we have

$$
E\left(Z^{k}\right)=\sum \frac{k !}{i_{1} ! \cdots i_{r} !} \frac{\Gamma\left(\sum_{j=1}^{r}\left(n_{j}+m_{j}\right)\right) \prod_{j=1}^{r} \Gamma\left(n_{j}+m_{j}+i_{j}\right)}{\prod_{j=1}^{r} \Gamma\left(n_{j}+m_{j}\right) \Gamma\left(\sum_{j=1}^{r}\left(n_{j}+m_{j}+i_{j}\right)\right)} E\left(X_{1}^{i_{1}}\right) \cdots E\left(X_{r}{ }^{i_{r}}\right),
$$

where $i_{1}+\cdots+i_{r}=k$. It is well known that, see [9],

$$
E\left(X_{j}{ }^{i}\right)=\frac{\Gamma\left(n_{j}+m_{j}\right) \Gamma\left(n_{j}+i_{j}\right)}{\Gamma\left(n_{j}\right) \Gamma\left(m_{j}+n_{j}+i_{j}\right)}
$$

for $j=1, \ldots, r$. So,

$$
\begin{aligned}
E\left(Z^{k}\right) & =\sum_{i_{1}+\cdots+i_{r}=k} \frac{k !}{i_{1} ! \cdots i_{r} !} \frac{\Gamma\left(\sum_{j=1}^{r}\left(n_{j}+m_{j}\right)\right) \prod_{j=1}^{r} \Gamma\left(n_{j}+m_{j}+i_{j}\right)}{\prod_{j=1}^{r} \Gamma\left(n_{j}+m_{j}\right) \Gamma\left(\sum_{j=1}^{r}\left(n_{j}+m_{j}+i_{j}\right)\right)} \prod_{j=1}^{r} \frac{\Gamma\left(n_{j}+m_{j}\right) \Gamma\left(n_{j}+i_{j}\right)}{\Gamma\left(n_{j}\right) \Gamma\left(m_{j}+n_{j}+i_{j}\right)} \\
& =\frac{\Gamma\left(\sum_{j=1}^{r}\left(n_{j}+m_{j}\right)\right)}{\Gamma\left(\sum_{j=1}^{r}\left(n_{j}+m_{j}+i_{j}\right)\right)} \sum_{i_{1}+\cdots+i_{r}=k} \frac{k !}{i_{1} ! \cdots i_{r} !} \prod_{j=1}^{r} \frac{\Gamma\left(n_{j}+i_{j}\right)}{\Gamma\left(n_{j}\right)} \\
& =\frac{\Gamma\left(\sum_{j=1}^{r}\left(n_{j}+m_{j}\right)\right)}{\Gamma\left(\sum_{j=1}^{r}\left(n_{j}+m_{j}+i_{j}\right)\right)} \sum_{i_{1}+\cdots+i_{r}=k}\left(\begin{array}{c}
k \\
i_{1}, i_{2}, \ldots, i_{r}
\end{array}\right) \frac{\Gamma\left(n_{1}+i_{1}\right)}{\Gamma\left(n_{1}\right)} \ldots \frac{\Gamma\left(n_{r}+i_{r}\right)}{\Gamma\left(n_{r}\right)} .
\end{aligned}
$$

By considering the fact that the sum of the Dirichlet-multinomial distribution on its support is equal to one (see [25]), we have

$$
E\left(Z^{k}\right)=\frac{\Gamma\left(\sum_{j=1}^{r}\left(n_{j}+m_{j}\right)\right)}{\Gamma\left(k+\sum_{j=1}^{r}\left(n_{j}+m_{j}\right)\right)} \frac{\Gamma\left(\sum_{j=1}^{r} n_{j}+k\right)}{\Gamma\left(\sum_{j=1}^{r} n_{j}\right)},
$$


which is the $k$ th moment of the $\operatorname{Beta}\left(\sum_{j=1}^{r} n_{j}, \sum_{j=1}^{r} m_{j}\right)$ distribution. Since $Z$ is a bounded random variable, its distribution is uniquely determined by its moments (Carleman's Theorem, see e.g. [12]).

In some cases our identification is indeed a characterization. Characterizations are of interest in many areas, see [10].

Theorem 2.3. Assume that the independent random variables $X_{1}, \ldots, X_{r}$ have common distribution on $(0,1)$ and the random vector $W=\left\langle W_{1}, \ldots, W_{r}\right\rangle$ has the Dirichlet $(2 \alpha, \ldots, 2 \alpha)$ distribution. Then $Z=\sum_{i=1}^{n} W_{i} X_{i}$ has a Beta $(r \alpha, r \alpha)$ distribution on $(0,1)$ if and only if $X_{1}, \ldots, X_{r}$ have the Beta $(\alpha, \alpha)$ distribution.

For the "if" part, put $n_{j}=\alpha, m_{j}=\alpha$ for $j=1, \ldots, r$ in Theorem 2.2.

For the "only if" part we prove by induction on $m$ that

$$
E\left(X_{j}^{m}\right)=\frac{\Gamma(m+\alpha) \Gamma(2 \alpha)}{\Gamma(\alpha) \Gamma(2 \alpha+m)} .
$$

- For $m=1$ we have

$$
E\left(X_{j}\right)=\frac{1}{2}
$$

- Now assume that for $k=1, \ldots, m$, we have

$$
E\left(X_{j}^{k}\right)=\frac{\Gamma(k+\alpha) \Gamma(2 \alpha)}{\Gamma(\alpha) \Gamma(2 \alpha+k)}
$$

and prove the desired conclusion for $m+1$.

We know that

$$
E\left(Z^{m+1}\right)=\sum_{i_{1}+\cdots+i_{r}=m+1}\left(\begin{array}{c}
m+1 \\
i_{1}, i_{2}, \ldots, i_{r}
\end{array}\right) E\left(W_{1}^{i_{1}} \cdots W_{r}^{i_{r}}\right) E\left(X_{1}^{i_{1}}\right) \cdots E\left(X_{r}^{i_{r}}\right)
$$

and

$$
\frac{\Gamma(m+1+r \alpha) \Gamma(2 r \alpha)}{\Gamma(r \alpha) \Gamma(2 r \alpha+m+1)}=\sum_{i_{1}+\cdots+i_{r}=m+1}\left(\begin{array}{c}
m+1 \\
i_{1}, i_{2}, \ldots, i_{r}
\end{array}\right)\left(\frac{\Gamma(2 r \alpha)}{\Gamma(2 r \alpha+m+1)} \prod_{j=1}^{r} \frac{\Gamma\left(2 \alpha+i_{j}\right)}{\Gamma(2 \alpha)}\right) \prod_{j=1}^{r} E\left(X_{i}^{i_{j}}\right) .
$$

So,

$$
\frac{\Gamma(m+1+r \alpha)}{\Gamma(r \alpha)}=\sum_{i_{1}+\cdots+i_{r}=m+1}\left(\begin{array}{c}
m+1 \\
i_{1}, i_{2}, \ldots, i_{r}
\end{array}\right) \prod_{j=1}^{r}\left(\frac{\Gamma\left(2 \alpha+i_{j}\right)}{\Gamma(2 \alpha)} E\left(X_{i}^{i_{j}}\right)\right)
$$

and

$$
\sum_{i_{1}+\cdots+i_{r}=m+1}^{i_{1}, \ldots, i_{r} \leqslant m} \frac{(m+1) !}{i_{1} ! \cdots i_{r} !} \prod_{j=1}^{r} \frac{\Gamma\left(2 \alpha+i_{j}\right)}{\Gamma(2 \alpha)} \frac{\Gamma(2 \alpha) \Gamma\left(\alpha+i_{j}\right)}{\Gamma(\alpha) \Gamma\left(2 \alpha+i_{j}\right)}+r \frac{\Gamma(2 \alpha+m+1)}{\Gamma(2 \alpha)} E\left(X_{i}^{m+1}\right)=\frac{\Gamma(r \alpha+m+1)}{\Gamma(r \alpha)} .
$$

Now, the proof is complete upon noting that

$$
\left(\frac{\Gamma(r \alpha+m+1)}{\Gamma(r \alpha)}-r \frac{\Gamma(\alpha+m+1)}{\Gamma(\alpha)}\right)+r \frac{\Gamma(2 \alpha+m+1)}{\Gamma(2 \alpha)} E\left(X_{i}^{m+1}\right)=\frac{\Gamma(r \alpha+m+1)}{\Gamma(r \alpha)} .
$$


Johnson and Kotz in the concluding remarks of their paper [16] mentioned that the "case in which the $X$ 's have standard uniform $(0,1)$ distribution leads to an interesting family of symmetric distributions." We identify it to be the celebrated beta distribution.

Example 2.1. For independent random variables $X_{1}, \ldots, X_{r}$ with standard uniform $(0,1)$ distributions and the random vector $W=\left\langle W_{1}, \ldots, W_{r}\right\rangle$ with the Dirichlet $(2, \ldots, 2)$ distribution, the random variable $Z=\sum_{j=1}^{r} W_{j} X_{j}$ has the Beta $(r, r)$ distribution. To see this it suffices to put $n_{j}=m_{j}=1$ for $j=1, \ldots, r$ in Theorem 2.2.

Example 2.2. Let $\left\{\alpha_{n}, \beta_{n}\right\}_{n=1}^{\infty}$ be a sequence of independent random variables and the random variables $\left\{X_{n}\right\}_{n=1}^{\infty}$ and $\left\{Y_{n}\right\}_{n=1}^{\infty}$ be defined inductively by

$$
\left(\begin{array}{cc}
X_{n} & 1-X_{n} \\
Y_{n} & 1-Y_{n}
\end{array}\right)=\left(\begin{array}{cc}
\alpha_{n} & 1-\alpha_{n} \\
\beta_{n} & 1-\beta_{n}
\end{array}\right)\left(\begin{array}{cc}
X_{n-1} & 1-X_{n-1} \\
Y_{n-1} & 1-Y_{n-1}
\end{array}\right), \quad n=2,3, \ldots
$$

with $X_{1}=\alpha_{1}$ and $Y_{1}=\beta_{1}$. Van Assche [24] showed that the limiting distributions of $X_{n}$ and $Y_{n}$ exist and are equal. [24, 17] reached the following equation: $\lim Y_{n}=\alpha_{1} Y^{*}+\beta_{1}\left(1-Y^{*}\right)$, where $\alpha_{1}, \beta_{1}, Y^{*}$ are all independent and $\alpha_{1}, \beta_{1}$ have the Beta $(a, a)$ distributions and $Y^{*}$ and $\lim Y_{n}$ have identical distributions (note that $Y^{*}$ depends on $\alpha_{n}$ 's and $\beta_{n}$ 's for $n \neq 1$ ). So, his results hold in our framework as well.

Theorem 2.4. Suppose $X_{1}, \ldots, X_{r}$ are independent random variables having the Beta $\left(\frac{1}{2}+\alpha_{1}, \frac{1}{2}+\alpha_{1}\right)$, $\ldots$, Beta $\left(\frac{1}{2}+\alpha_{r}, \frac{1}{2}+\alpha_{r}\right)$ distributions and that the random vector $W=\left\langle W_{1}, \ldots, W_{r}\right\rangle$ has the Dirichlet $\left(\alpha_{1}, \ldots, \alpha_{r}\right)$ distribution. Then $Z=\sum_{j=1}^{r} W_{j} X_{j}$ has the Dirichlet $\left(\frac{1}{2}+\sum_{i=1}^{r} \alpha_{i}, \ldots\right.$, $\left.\frac{1}{2}+\sum_{i=1}^{r} \alpha_{i}\right)$ distribution.

Let $Y_{j}(j=1, \ldots, n)$ be independent random variables independent from $X_{1}, \ldots, X_{n}$ that have the $\operatorname{Gamma}\left(\alpha_{j}\right)$ distribution. It can be seen, by some classical ways (e.g. $\left.E\left(e^{t^{\prime} T}\right)=[\Psi(t)]^{\sum_{j} \alpha_{j}}\right)$ from [18, Table 2]), that the distribution of $T=\sum_{j} T_{j}=\sum_{j} Y_{j} X_{j}$ is the same as the distribution of $T_{j}$ with the parameter $\sum_{j} \alpha_{j}$. We can also write $T$ as

$$
T=\sum_{j} Y_{j} X_{j}=\left(\sum_{i} Y_{i}\right)\left(\sum_{j} \frac{Y_{j}}{\sum_{i} Y_{i}} X_{j}\right)
$$

and so we have $T=Y Z$ in which $Y$ has the gamma distribution with the parameter $\sum_{j} \alpha_{j}$, and $T$ has the $F$ distribution with the parameter $\sum_{j} \alpha_{j}$, and $Y$ and $Z$ are independent from each other. Of course, one can define $T^{\prime}=Y^{\prime} X^{\prime}$ in such a way that $T^{\prime} \stackrel{d}{=} T, \sum_{j} Y_{j} \stackrel{d}{=} Y^{\prime}$ and $X^{\prime} \sim$ Dirichlet $\left(\frac{1}{2}+\sum_{j} \alpha_{j}\right)$. One can conclude that $Z$ and $Z^{\prime}$ have identical distributions by calculating the general moments $\left(s_{1}, s_{2}\right)$ of $T$ and $T^{\prime}$, i.e., $E\left(Z_{1}^{s_{1}} Z_{2}^{s_{2}}\right)=E\left(\left(X_{1}^{\prime}\right)^{s_{1}}\left(X_{2}^{\prime}\right)^{s_{2}}\right)$.

\section{An approximation}

In this section, we propose an approximation for the distribution of $Z=\sum_{j=1}^{r} W_{j} X_{j}$. By Theorem 2.1 and the fact that the support of $Z$ lies in the interval $[0,1]$, we are interested in approximating its distribution by a suitable member of the two-parameter $\operatorname{Beta}(p, q)$ family of distributions. The idea of approximating distributions having complicated formulas by the beta distribution is 
very well established in the statistics literature, see e.g. [13], [14] and [19]. Following these authors, the choice of the beta parameters $p$ and $q$ is made using the method of moments. Equating the first two moments of $Z$ with those of the beta distribution, we infer that

$$
E(Z)=\frac{p}{p+q},
$$

and

$$
E\left(Z^{2}\right)=\frac{p(p+1)}{(p+q)(p+q+1)}
$$

which we must solve simultaneously to find the beta parameters $p$ and $q$. After some algebraic manipulation, we find the solutions as

$$
p=\frac{E(Z)-E\left(Z^{2}\right)}{\operatorname{Var}(Z)} E(Z)
$$

and

$$
q=\frac{E(Z)-E\left(Z^{2}\right)}{\operatorname{Var}(Z)}[1-E(Z)]
$$

Note that the above solutions satisfy $p>0$ and $q>0$. The two moments $E(Z)$ and $E\left(Z^{2}\right)$ can be computed by Theorem 2.3.

The robustness of this approximation was checked for a range of values of the parameters. The following procedure based on simulation and the Kolmogorov-Smirnov test was used:

step 1 - For the given parameters, compute the $E(Z)$ and $E\left(Z^{2}\right)$.

step 2 - Obtain the corresponding estimates for $(p, q)$.

step 3 - Simulate 1000 random numbers of $Z=\sum_{j=1}^{r} W_{j} X_{j}$ by simulating the $X_{i}$ 's and $W_{i}$ 's.

step 4 - Perform the Kolmogorov-Smirnov test to see whether the sample in step 3 arises from the beta distribution with parameters $p$ and $q$ calculated in step 2 .

The $p$-values of this test for the approximation are given in Tables 2 and 3 . These tables show that approximation is robust for most of the chosen parameters. Similarly the distribution of $T$ can be approximated.

As an example, suppose that $W, X_{1}, X_{2}$ are independent and all have the uniform $[0,1]$ distribution. The probability density function (PDF) of $Z$ is $f_{Z}(z)=-2(1-z) \log (1-z)-2 z \log z$, $0<z<1$. According to the first row of Table $2, B(1.75,1.75)$ will be a good approximation for $Z$. Figure 1 shows the exact and approximate PDFs of $Z$. 


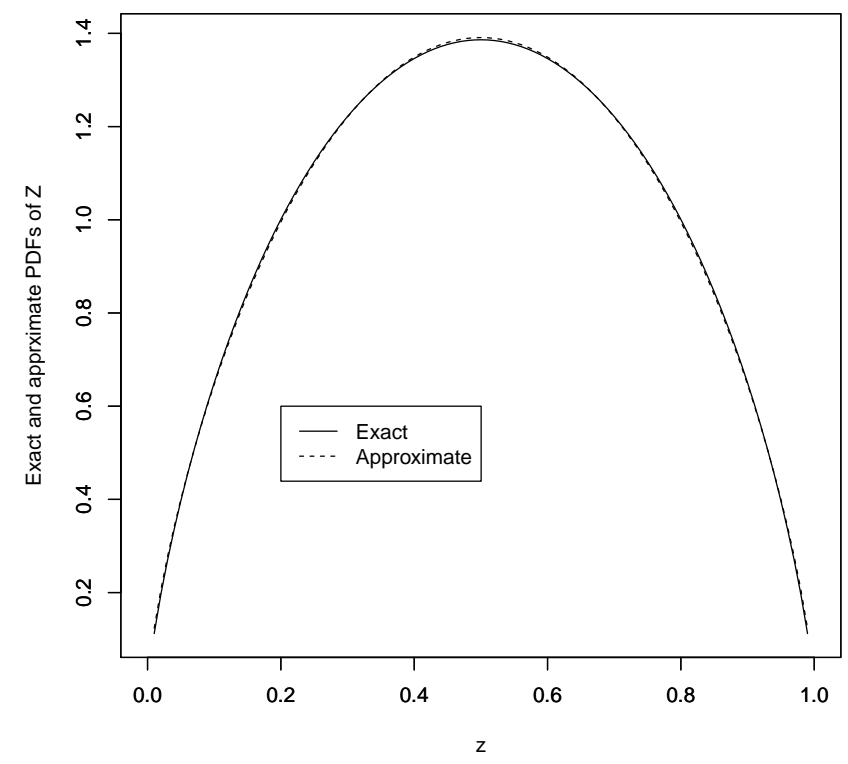

Figure 1: The exact and approximate PDFs of $Z$ when $W, X_{1}$ and $X_{2}$ are uniform. The approximate distribution is $\mathrm{B}(1.75,1.75)$.

The error in Euclidean norm of this approximation is

$$
\|f-B(1.75,1.75)\|_{2}=\left\{\int_{0}^{1}[f(z)-B(1.75,1.75)]^{2} d z\right\}^{\frac{1}{2}}=0.0045 .
$$

\section{Distribution of lifetime in the environment independent $r$-position}

Here, we identify the distribution of sums of the random variables introduced in [21]. Let us recall that identifying this distribution by direct means can be very difficult.

Theorem 4.1. Suppose $X_{1}, \ldots, X_{r}$ are independent random variables with the $\operatorname{Beta}\left(n_{1}, n-n_{1}\right)$, $\ldots, \operatorname{Beta}\left(n_{r}, n-n_{r}\right)$ distributions. Suppose $Y_{1}, \ldots, Y_{r}$ are independent random variables with the Gamma $\left(n, \frac{1}{\theta}\right)$ distributions independent from $X_{i}$ 's. Then the distribution of $T=\sum_{i=1}^{r} X_{i} Y_{i}$ is equal to

$$
f(t)=\frac{\theta^{-r n} \Gamma\left(r-\sum_{j=1}^{r} n_{j}\right)}{\Gamma(r n) B\left(\sum_{j=1}^{r} n_{j}, r-\sum_{j=1}^{r} n_{j}\right)} z^{r n-1} e^{-\frac{t}{\theta}} \Psi\left(r-\sum_{j=1}^{r} n_{j}, 1+n-\sum_{j=1}^{r} n_{j} ; \frac{t}{\theta}\right),
$$

where

$$
\Psi(a, b ; x)=\frac{1}{\Gamma(a)} \int_{0}^{\infty} e^{-x u} u^{a-1}(1+u)^{b-a-1} d u
$$


Let $Y_{+}=\sum_{i=1}^{r} Y_{i}$ and write

$$
T=Y_{+} \sum_{i=1}^{r} X_{i} \frac{Y_{i}}{Y_{+}}
$$

It can be clearly seen that $Y_{+}$and $Y_{i} / Y_{+}$'s are independent from each other. On the other hand the vector $\left\langle Y_{1} / Y_{+}, \ldots, Y_{r} / Y_{+}\right\rangle$has the Dirichlet $(n, \ldots, n)$ distribution. So, $T$ is distributed as the product of two independent random variables one of which has the gamma distribution and the other one has the beta distribution by Theorem 2.2. Hence, the theorem is proved by Theorem 1 of $[21]$.

Corollary 4.1. Suppose $X_{1}, \ldots, X_{r}$ are independent random variables with the Beta $\left(n_{1}, n-n_{1}\right)$, $\ldots, \operatorname{Beta}\left(n_{r}, n-n_{r}\right)$ distributions. Suppose $Y_{1}, \ldots, Y_{r}$ are independent random variables with the Gamma $\left(n, \frac{1}{\theta}\right)$ distributions and also independent from $X_{i}$ 's. Then the distribution of $\bar{T}$ is equal to

$$
f_{\bar{T}}(t)=\frac{\theta^{-r n} \Gamma\left(r-\sum_{j=1}^{r} n_{j}\right)}{\Gamma(r n) B\left(\sum_{j=1}^{r} n_{j}, r-\sum_{j=1}^{r} n_{j}\right)} z^{r n-1} e^{-\frac{r t}{\theta}} \Psi\left(r-\sum_{j=1}^{r} n_{j}, 1+n-\sum_{j=1}^{r} n_{j} ; \frac{r t}{\theta}\right)
$$

where

$$
\Psi(a, b ; x)=\frac{1}{\Gamma(a)} \int_{0}^{\infty} e^{-x u} u^{a-1}(1+u)^{b-a-1} d u .
$$

Theorem 4.2. Suppose $X_{1}, \ldots, X_{r}$ are independent and identical random variables distributed over $(0,1)$ and $Y_{1}, \ldots, Y_{r}$ are independent exponential random variables with the mean $\theta$ and also independent from $X_{j}$ 's. Then $T=\sum_{j=1}^{r} X_{j} Y_{j}$ has the Gamma $\left(r \alpha, \frac{1}{\theta}\right)$ distribution if and only if $X_{j}$ 's have the Beta $(r \alpha, r(1-\alpha))$ distributions.

For the "if" part, it suffices to find the distribution of $X_{j} Y_{j}$.

For the "only if" part, we have $T=Y_{+}\left(\sum_{i=1}^{n} \frac{Y_{i}}{Y_{+}} X_{i}\right)$, where $Y_{+}=\sum_{i=1}^{r} Y_{i}$. Theorem 1 of [27] implies that $\sum_{i=1}^{n} \frac{Y_{i}}{Y_{+}} X_{i}$ should have the beta distribution; now Theorem 2.2 completes the proof.

Using the techniques of the above theorems we can give an alternative proof for the main theorem:

[A second proof for Theorem 2.2] Suppose that $\left\{X_{i}\right\}_{i=1}^{n}$ are independent random variables with the $\left\{\operatorname{Beta}\left(\alpha_{i}, \beta_{i}\right)\right\}_{i=1}^{n}$ distributions, and $\left\{Y_{i}\right\}_{i=1}^{n}$ are independent gamma random variables with parameters $\left\{\alpha_{i}+\beta_{i}\right\}_{i=1}^{n}$; also $X_{i}^{\prime}$ 's and $Y_{i}^{\prime}$ 's are independent from each other. Let

$$
Y_{+}=\sum_{i=1}^{n} Y_{i} \quad \text { and } \quad T=Y_{+}\left(\sum_{i=1}^{n} \frac{Y_{i}}{Y_{+}} X_{i}\right)
$$

By Theorem 1 of [27], since $Y_{+}$has the Gamma $\left(\sum_{i=1}^{n} \alpha_{i}+\sum_{i=1}^{n} \beta_{i}\right)$ distribution and $T$ has the $\operatorname{Gamma}\left(\sum_{i=1}^{n} \alpha_{i}\right)$ distribution, $\sum_{i=1}^{n} \frac{Y_{i}}{Y_{+}} X_{i}$ has the Beta $\left(\sum_{i=1}^{n} \alpha_{i}, \sum_{i=1}^{n} \beta_{i}\right)$ distribution. The theorem follows by noting that $\left\langle\frac{Y_{1}}{Y_{+}}, \ldots, \frac{Y_{n}}{Y_{+}}\right\rangle$has the Dirichlet $\left(\alpha_{1}+\beta_{1}, \alpha_{2}+\beta_{2}, \ldots, \alpha_{n}+\beta_{n}\right)$ distribution.

The following theorem can be proved similarly to Theorem 4.1. 
Theorem 4.3. Suppose $X_{1}, \ldots, X_{r}$ are independent random variables with the Beta $\left(n_{1}, m_{1}\right)$, $\ldots$, Beta $\left(n_{r}, m_{r}\right)$ distributions and $Y_{1}, \ldots, Y_{r}$ are independent random variables with the Gamma $\left(\alpha_{i}, \frac{1}{\theta}\right)$ distributions $(i=1, \ldots, r)$ which are independent from $X_{i}$ 's. Then we have $T \stackrel{d}{=} Y Z$ for $a$ gamma random variable $Y$.

Therefore, $T$ can be obtained by using Theorems 4.3 and 4.1 .

\section{Conclusions}

We have studied the distribution of the mixture random variable $Z$ (and $T$ ) when the $X_{i}$ 's have beta distributions. It is interesting to note that the distribution of $Z$ can be the beta distribution, and the distribution of $T$ can be that introduced in [21]. Both these distributions have been studied with applications in [21]. It seems, by the results obtained in this paper, the environment does not essentially change the distribution; only its parameters. It would be interesting to study the multivariate cases of these results.

\section{References}

[1] Abourizk, S.M., Halpin, D.W., Wilson, J.R. (1994). Fitting Beta Distributions Based on Sample Data, Journal of Construction Engineering and Management 120, 288-305.

[2] Alamatsaz, M.H. (1985). A Note on An Article by Artikis, Acta Mathematica Hungarica 45, 159-162.

[3] Alamatsaz, M.H. (1993). On Characterizations of Exponential and Gamma Distributions, Statistics and Probability Letters 17, 315-319.

[4] Alamatsaz, M.H., Nekoukhou, V. (2012). A Family of Skew-Symmetric-Laplace Distributions, Statistical Papers 53, 685-696.

[5] Alamatsaz, M.H., Rezapour, M. (2014). Stochastic Comparison of Lifetimes of Two ( $n-$ $k+1$ )-out-of- $n$ Systems with Heterogeneous Dependent Components, Journal of Multivariate Analysis 130, 240-251.

[6] Asadi, M. (1998). Characterization of the Pearson System of Distributions Based on Reliability Measures, Statistical Papers 39, 347-360.

[7] Asadi, M. (2016). Jensen-Shannon Information of the Coherent System Lifetime, Reliability Engineering and System Safety 156, 244-255.

[8] Asadi, M., Goli, S. (2017). A Study on the Conditional Inactivity Time of Coherent Systems, Metrika 80, 227-241.

[9] Balakrishnan, N., Nevzorov, V.B. (2003). A Primer on Statistical Distributions, John Wiley and Sons, New York.

[10] Behboodian, J. (1989). Symmetric Sum and Symmetric Product of Two Independent Random Variables, Journal of Theoretical Probability 2, 267-270. 
[11] Beutner, E., Kamps, U. (2009). Identical Distributions of Single Variates and Random Convex Combinations of Uniform Fractional Order Statistics, Communications in Statistics-Theory and Methods 38, 1950-1959.

[12] Chung, K.L. (2001). A Course in Probability Theory, third edition, Academic Press, New York.

[13] Fan, D.Y. (1991). The Distribution of the Product of Independent Beta Variables, Communications in Statistics-Theory and Methods 20, 4043-4052.

[14] Gupta, A.K., Nadarajah, S. (2006). Exact and Approximate Distributions for the Linear Combination of Inverted Dirichlet Components, Journal of the Japan Statistical Society 36, $225-236$.

[15] Gupta, A.K., Nadarajah, S. (2008). Normal and Logistic Random Variables: Distribution of the Linear Combinations, Statistical Papers 49, 201-209.

[16] Johnson, N.L., Kotz, S. (1990). Randomly Weighted Averages: Some Aspects and Extensions, The American Statistician 44, 245-249.

[17] Johnson, N.L., Kotz, S. (1990). Use of Moments in Deriving Distributions and Some Characterizations, The Mathematical Scientist 15, 42-52.

[18] Kerov, S.V.E., Tsilevich, N.V. (2004). The Markov-Krein Correspondence in Several Dimensions, Journal of Mathematical Sciences 121, 2345-2359.

[19] Nadarajah, S. (2006). Exact and Approximate Distributions for the Product of Inverted Dirichlet Components, Statistical Papers 47, 551-568.

[20] Nadarajah, S., Cordeiro, G.M., Ortega, E.M.M. (2013). The Exponentiated Weibull Distribution: A Survey, Statistical Papers 54, 839-877.

[21] Nadarajah, S., Kotz, S. (2005). On the Product and Ratio of Gamma and Beta Random Variables, Allgemeines Statistisches Archiv 89, 435-449.

[22] Pham-Gia, T., Turkkan, N. (1994). Reliability of a Standby System with Beta-Distributed Component Lives, IEEE Transactions on Reliability 43, 71-75.

[23] Shakil, M., Kibria, B.M.G. (2009). Exact Distributions of the Linear Combination of Gamma and Rayleigh Random Variables, Austrian Journal of Statistics 38, 33-44.

[24] Van Assche, W. (1986). Products $2 \times 2$ Stochastics Matrices with Random Entries, Journal of Applied Probability 23, 1019-1029.

[25] Wang, K.N., Tian, G.L., Tang, M.L. (2011). Dirichlet and Related Distributions: Theory, Methods and Applications, John Wiley and Sons, New York.

[26] Wilks, S.S. (1962). Mathematical Statistics, John Wiley and Sons, New York.

[27] Yeo, G.F., Milne, R.K. (1989). On Characterizations of Exponential Distributions, Statistics and Probability Letters 7, 303-305. 